\title{
LTE BASED VEHICLE TRACKING AND ANTI-THEFT SYSTEM USING RASPBERRY PI MICROCONTROLLER
}

\author{
Dina A. Bahr ${ }^{1}$, Osama A. Awad ${ }^{2}$ \\ 1,2 College of Information Engineering, Al-Nahrain University, Baghdad, Iraq \\ \{dina.abdullah ${ }^{1}$, usamaawad $\left.{ }^{2}\right\} @$ coie-nahrain.edu.iq \\ Received:18/1/2019, Accepted:4/3/2019
}

\begin{abstract}
This paper is dealing with the implementation and design of an integrated anti-theft control system for an automobile, also to prevent vehicles from stealing. This embedded system mainly based on an advanced communication technology identified as LTE Release 8 or 3.9 G. The factors that associated with system structured are putting together inside the vehicle in a hidden way. A GSM module has a SIM-Card interface that responsible for exchanging messages with end-user to track and control the status of the vehicle. Once the vehicle is stolen, firstly, a phone calling from the owner is initiated to the GSM. Immediately the RPi cut-off this call and activate GPS respectively with Pi Camera activation, the location coordinates of a vehicle are sent as SMS via GSM module to the owner, as soon as a Pi Camera capture a photo for the thief that will be sent later on to owner via advance internet services. A Google map applied the received coordinate to determine the vehicle movement. According to these instructions, the owner sends SMS that has a detailed instruction to order RPi to control the engine ignition system by turning it off at a required location. The principal approach of this embedded system has been designed and executed to be effectively at low price. Furthermore; administering subsequent various on-road tests demonstrated to be very powerful in controlling stolen vehicles.
\end{abstract}

\section{keywords: LTE Release8,3.9G, GSM-GPRS SIM900A, GPS, Raspberry Pi, PiCamera.}

\section{INTRODUCTION}

The security circumstances have been improved in all domains of modern generations. Furthermore, vehicle security must encompass several rapid developments. The cost of overall security restructures are expensive. Nowadays, the automotive industry develops more sophisticated with the evolution of modern technology. the community is increasingly concerned with preventing the theft of their vehicle. Accordingly, modern companies are attempting to produce an advanced antitheft system[1]. The system is characterized as a technology that is employed to obtain the location of a vehicle utilize technologies such as GPS, GSM and other radio navigation systems that are handling between satellites and ground stations. So, manipulation with transportation services and modern applications that are provided by communication services to developing the purpose of the anti- theft system [2]. Broadly, products of vehicle anti- theft devices technologically categorized into three classes: vehicle tracking and recovery systems, the alarm system for a car and devices that lock a vehicle mechanically [3]. Our project is trying to design better-guaranteed security solutions to the vehicle with a credible cost. The system is designed to be simple and with least expenditures. The Raspberry Pi represents the heart of the system. Also exploited the facilities of GPS to tracking vehicle over Google map. Accordingly, the SIM card is determined over a GSM with 3.9G LTE Release 8 service that is provided by the communication authority of the Iraqi government. Furthermore, the details about the stealing accident are generated and reported automatically to send via email services to a local police authority. This paper is arranged in the following sequence: The current and most important works of the literature survey are presented with the problem statement that demonstrated in the next section. In section three, we proposed the idea of our system. Overall system structure is presented in section four. The Raspberry Pi hardware and software components used in the design are presented in section 5. Advanced communications related to the proposed 
system is explained in section six. The methodology, hardware circuits, and software running are explained in section 7. The results of tracking and controlling system are figured out in section 8. Finally, the conclusions drawn from this work is given.

\section{Literature ReVIEW}

The conceptual framework for the most related and current researches are presented. Most importing flexibility between the communication field and raspberry pi has been achieved yet. Lee et al in 2014 [4] designed an attractive tracking system with wireless data transportation (i.e.GSM \& SMS technology). Exploitation of SMS technology over GSM network offers the user the vehicle location report. The position of the vehicle is displayed on Google maps. Another an efficient tracking system has proposed to detecting vehicle motion, depending on the embedded Linux board particularly Raspberry Pi with a service of android on Smartphone that works on a GPS/ GSM/ GPRS module by using a SIM900A. This system put inside the vehicle whose location will be determined on a web page in real time. Here Android smartphone of the owner will determine the required root that the vehicle must follow. The specified path will be matched with the current driver path. Once, if the driver carries the vehicle on the wrong path, an alert message will be sent to the owner, as achieved by Shinde, et. al. in 2015 [5]. In the same year, Ajish. et. al. [6] acquaints a low-cost extendable structure its dependence face detection subsystem. The system automatically takes images for the driver and then compares this photo with a database. This system mainly based on an optimized principle component analysis (PCA) algorithm. In most, the recent year 2017 Vaibhav, et al [7] proposed an automobile protection system by applying an embedded system with a Wi-Fi module and GPS. dealing with investigated the stolen vehicle and accidents that are happening. The system is meant to send predefined messages if the vehicle gets stolen and if calamity to vehicle happens. The owner track vehicle position in real time using GPS locator. The vehicle real-time location will be sent via the internet Wi-Fi. A real-time tracking system is employed by Mohammad, et., al in 2016[8] with GPS. The proposed framework makes powerful utilization of innovation that deals with Arduino Intel Galileo as an embedded board microcontroller. This system introduces GSM-GPRS GPS and applying SIM908. A PHP webpage is designed to load Google maps which responsible for browsing the locations in real-time. In the same scenario, the image processing field was discussed by Anandhalli and Baligar in 2017[9], they are present a process algorithmic rule for a video image that tracks, detects, and counts the number of cars on a street. It works on RGB frame of videos that pander to the conversion of RGB to HSV color domain, the detection of cars is solely carrying on color properties of cars that may facilitate in distinctive the colors of cars entirely. The noise drawback in every frame was removed. trailing of the automobile is completed by victimization the Kalman filter with an information assortment. this method can verify the number of cars that are enforced within the video or on a selected path. the concept of this novel is to trace, count and sight cars on the road and it's been running on Raspberry Pi 3 and by victimization C++ and OpenCV. In the objects of restrictions that the presenting studies might suffer from two principal subjects that could be outlined. first of all, there was no application accustomed stop the vehicle remotely through GSM network. Secondly, the analysis attended specialize in tracking the stolen vehicle in a specific location so it failed to cover the entire target of the anti-theft system. According to these aforementioned determinations this paper will accomplish and propose a solution for the main problems statement that summarized by the following :

https://ijict.edu.iq 
- The main issue of the anti-theft vehicle tracking system is how to design an integrated advanced system with costeffective innovative technology to assist people to stop a stolen vehicle at anywhere they want.

- Identify the thief by analyzing and recognizing from a captured photo taken by a PiCamera module of the embedded system. This information should be passed to the owner via an advanced communication technology that is using the internet of LTE Release8 services. The evaluation system will be helpful to ensure their safety at all the time.

\section{System Proposal}

The principal intention of our study is to minimize the theft of vehicles. Here we are implementing a standalone embedded system based on Linux OS and ARM11 processor using Raspberry P3 B. This project will design and implemented to solve the problem of vehicle's theft and tracking with full control to its motion. So when the vehicle was stolen, RPi orders a relay to stop the engine according to owner premise. At the same time, PiCamera and GPS are activated to capture a driver's photo and track the vehicle's location on the Google map. The service $3.9 \mathrm{G}$ is exploiting to provide a high speed $\&$ better quality for transmitting the image over a communication media. In addition, an email report automatically sent to a police station. This system is appropriately suitable for all versions of vehicles.

\section{SySTEM STRUCTURE}

The design of the proposed system is summarized by the block diagram as shown in Fig. 1 A Raspberry Pi, GSM/GPRS SIM900A Module, GPS Module, Pi Camera, Relay, Fuse of the fuel pump, Power supplied and other important equipment blocks which interact together to perform the proposed system. When the vehicle is turned on, a power supply applied $5 \mathrm{~V}$ from vehicles' battery to the RPi and $12 \mathrm{~V}$ must be supplied to relay inside a vehicle. Only authorized personnel know a secret number of the GSM module that setting secretly inside the vehicle. When the vehicle is stolen, the owner creates a phone call to the GSM, Raspberry pi rejects a call and then send vehicle location and photo as SMS and MMS respectively. After that owner sends an SMS to stop the vehicle engine at a specified location[10].

\section{Raspberry Pi Hardware and Software Components}

Recently, the evaluation of Raspberry Pi microcontroller shows a high potential of unlocked computing that applied in a vast number of areas. The advantages of the RPi is lead to produce a unique system with full characterization.

\section{A. Raspberry Pi Hardware Concepts}

The Microcontroller represents the heart of the project since it controls the devices being interfaced with it, and let them communicate with each other through this controller according to the program written in. It contains a Broadcom BCM2835 that is representing system on a chip. The processor is running at $700 \mathrm{MHz}$ and structured by familiar CPU of the microarchitectures implementation of the ARM Cortex/A53. Raspberry Pi running upon (802.11n) Wireless LAN according to the manufacturing industry instructions [11]. RPi 3 model B is having onboard USB capabilities, Bluetooth and Wi-Fi. This little microcontroller is smart and enables the educator to explore the computing and programming languages like C++, Java, Python, $\mathrm{C}$ and Scratch that installation on it by default [12]. Python is the language used in programming 


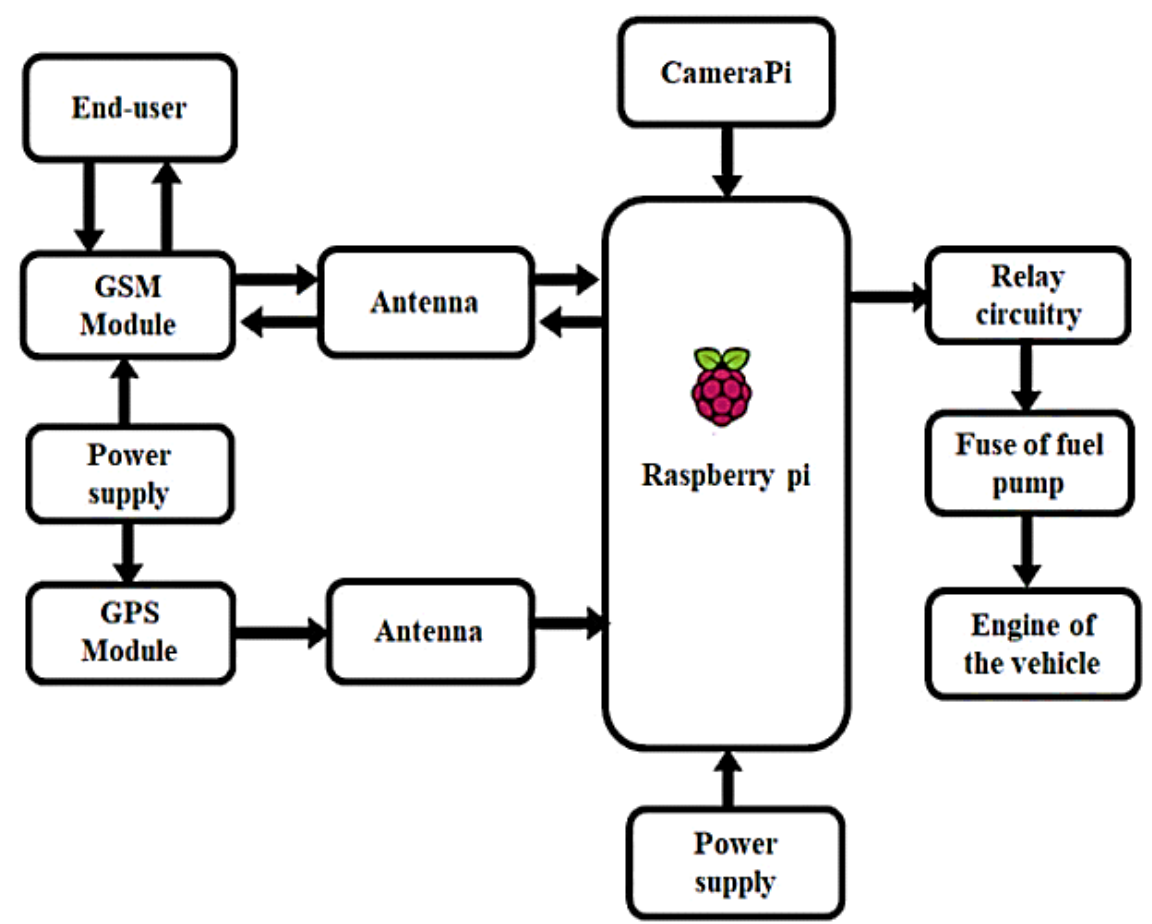

Figure 1: Block diagram for the proposed system

all the modules in this work. In the main structure of the Broadcom is GPIO that permit data to follow automatically and producing a system with digital control programming setting. GPIO module allows the interfacing with any electronic device to become simplistic[8].

\section{B. Raspberry Pi Software Concepts}

The Raspberry Pi software programming identified as Raspbian running upon Linux distribution representing a Debianbased for downloading OS. Raspbian programming provides a different language and taken a Python and Scratch as fundamental languages that important and powerful to learn by the educator[8]. Raspbian program running with a high performance that originates with up to (35,000 packages), so to get access to RPi operating system, must instilling Putty Software on windows or enable VNC viewer[13].

\section{PiCamera Module}

An interesting hardware device on top of the board of the RPi represented as a tiny circuit that perfectly printed on the board, the small camera identified as PiCamera. The connecting between RPi and PiCamera via a ribbon cable through a hub of RPi hardware. The resolution of PiCamera is (5MP)[13]. PiCamera required enabling from the Raspbian configuration setting. Furthermore, to capture image write a program in form of Python script [8]. 


\section{Vi. Advanced Communications Facilities}

Cellular systems previously built around the circuit-switched model that represents the only model over generations of communication technology. The term of a Long Term Evolution, that is recently improved to support packet-switched services. Furthermore, Internet protocol (IP) supported the smooth connection between a packet data network (PDN) and the user equipment (UE) that prevent any difficulties in the applications of the end-users during mobility. However, the LTE covers the development of the radio access of the Universal Mobile Telecommunications System (UMTS) through the enhanced UTRAN (E-UTRAN). This accompanied with the growth of a non-radio portion comes under the name of System Architecture Evolution (SAE) [14]. The set of requirements, regarding the environment to achieve minimum peak data rates for outdoor where the data rate equals $384 \mathrm{kbps}$ and the indoor reaches $2048 \mathrm{kbps}$ was introduced [16].

\section{A. Multiple Access for LTE Concept}

$4 \mathrm{G}$ wireless communication developed successfully from the international telecommunications union radio communication sector (ITU-R). 3GPP Release 8 included LTE for mobile broadband services. The industry of wireless focuses on providing optimized packet, low-latency, highly efficient and more secure services. The radio access increases the power of downlink transmission by modulating waveforms in Orthogonal Frequency Division Multiplexing. The reason behind using the OFDM is to prevent the inter-symbol interference which leads to limit the system's performance for high-speed parameter and for a peak-to-average power ratio (PAPR) which tightening the uplink with convenient SC-FDMA properties. In parallel, the techniques of MIMO (Multiple-Input Multiple-Output) to support a better data rate is applied. The OFDMA spacing of subcarrier is $(15 \mathrm{kHz})$ in the bandwidth of the transmission signal. Various subcarriers must be maintained orthogonally, the sampling instant of one subcarrier signal to another subcarrier signal must equal to zero value[16], as shown in Fig. 2. The

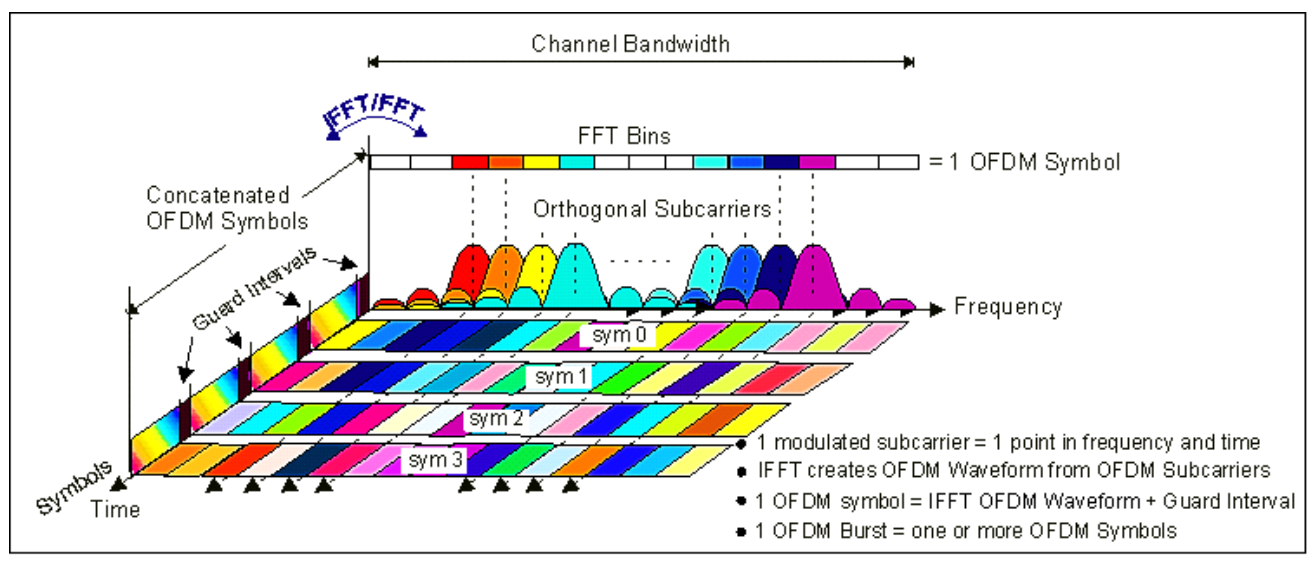

Frequency-Time Representative of an OFDM signal

Figure 2: Generation frequency domain of OFDMA [14]

Fast Fourier Transform (FFT) is using to change between a time and frequency domain. The structure of the transmitter is quite simple that represents a basic form for SC-FDMA including a cyclic prefix in Fig. 3. 


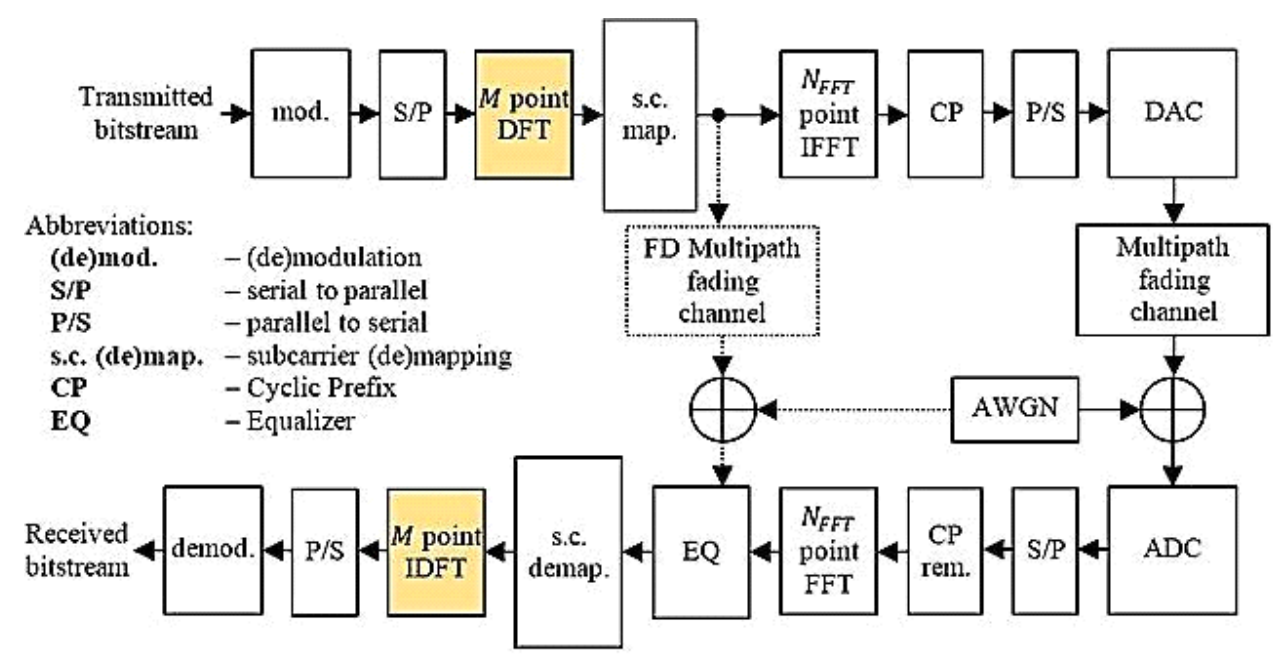

Figure 3: Generation frequency domain of SC-FDM[15]

\section{B. GSM Module Studio}

A second-generation digital network over local-area is distributed[17]. The most differentiation properties from other generations are the speech and signaling channels work in a digital form. Mainly, GSM dependent on TDMA[2]. The GSM cellular system transmits data over the rates that equal to $271 \mathrm{kbps}$ upon transmission bandwidth is equal to 200 $\mathrm{kHz}$. This structure provides an effect that double the bit rate per subscriber at allocating just one slot per frame[17]. The module is supported with SIM900A that represent a complete Dual-band GSM/GPRS solving in an SMT module that has an allocating frequency band between $1800 \mathrm{MHz}$ and $1900 \mathrm{MHz}$ and delivers with high performance for voice, SMS, Data, and Fax with low power consumption. The protocols of UART have a baud rate of 115200 bps with SIM card reader via an interface connector with internal TCP/IP stack to enable you to connect with internet via GPRS[14].

\section{GPS Module}

The GPS system covering the earth with 24 satellites orbiting and applied for the position with the information's time in any statute of the weathered. The GPS receiver gives $-157 \mathrm{dBm}$. GPS received digital signals of satellite devices and sent to an RPi. The signals represent the coordinates in form of the latitudes, longitudes, and altitudes[18]. To calculate a specific region inside the satellite orbits,suppose that"vehicle moves on the street", and the region of center on earth as the original region in the coordinate of the system that works within three dimensions. The system needed at least four satellites in view to provide information about the vehicle's position. The satellites represented as (S1, S2, S3, and S4), Now suggest that $\mathrm{Si}$ is exited at $(\mathrm{Xi}, \mathrm{Yi}, \mathrm{Zi})$ which transmit a signal with a time Ti. So, if the time of the signal is received at Ti', by depending on the time in specification receiver, the equation that is used to calculate the time been $\left(\Delta T_{i}=T_{i}{ }^{\prime}-T i\right)$. Where the symbol $(\varepsilon)$ represent any error occurs in the clock's time. The distances $d\left(\Delta T_{i}, \varepsilon\right)$ computed by equation (1) 
refers to how the satellites are far from the specific location on the earth [19] :

$$
\begin{aligned}
& \left(x_{0}-X_{1}\right)^{2}+\left(y_{0}-Y_{1}\right)^{2}+\left(z_{0}-Z_{1}\right)^{2}=d\left(\Delta t_{1}, \varepsilon\right)^{2} \\
& \left(x_{0}-X_{2}\right)^{2}+\left(y_{0}-Y_{2}\right)^{2}+\left(z_{0}-Z_{2}\right)^{2}=d\left(\Delta t_{2}, \varepsilon\right)^{2} \\
& \left(x_{0}-X_{3}\right)^{2}+\left(y_{0}-Y_{3}\right)^{2}+\left(z_{0}-Z_{3}\right)^{2}=d\left(\Delta t_{3}, \varepsilon\right)^{2} \\
& \left(x_{0}-X_{4}\right)^{2}+\left(y_{0}-Y_{4}\right)^{2}+\left(z_{0}-Z_{4}\right)^{2}=d\left(\Delta t_{4}, \varepsilon\right)^{2}
\end{aligned}
$$

Where $\left(X_{i}, Y_{i}, a n d Z_{i}\right)$ are the rectangular coordinates of the satellites for $(\mathrm{i}=1, . .4)$. The rectangular coordinates $\left(x_{0}, y_{0}\right.$, and $\left.z_{0}\right)$ for the receiver are converted after solution to represent the coordinates spherically to latitude, longitude, and altitude over sea level. While d refers to the difference in time between the receiver and the satellite's clocks, and ( $t$ ) is the travel time for the signal from the satellite to the receiver and (c) is the speed of light in space $\left(3 \times 10^{8} \mathrm{~km} / \mathrm{s}\right)$. Fig. 4 below show the GPS receiver with four satellites on view. We summarized the procedure that demonstrated an approximation of the receiver's location by solving the system equations for specific satellite coordinates using the (quadratic equation and Newton's method). We will then demonstrate sources for error in the approximation by choosing different satellite positions and amounts, where the GPS module arranges all these mathematical equations over the python programming packages.

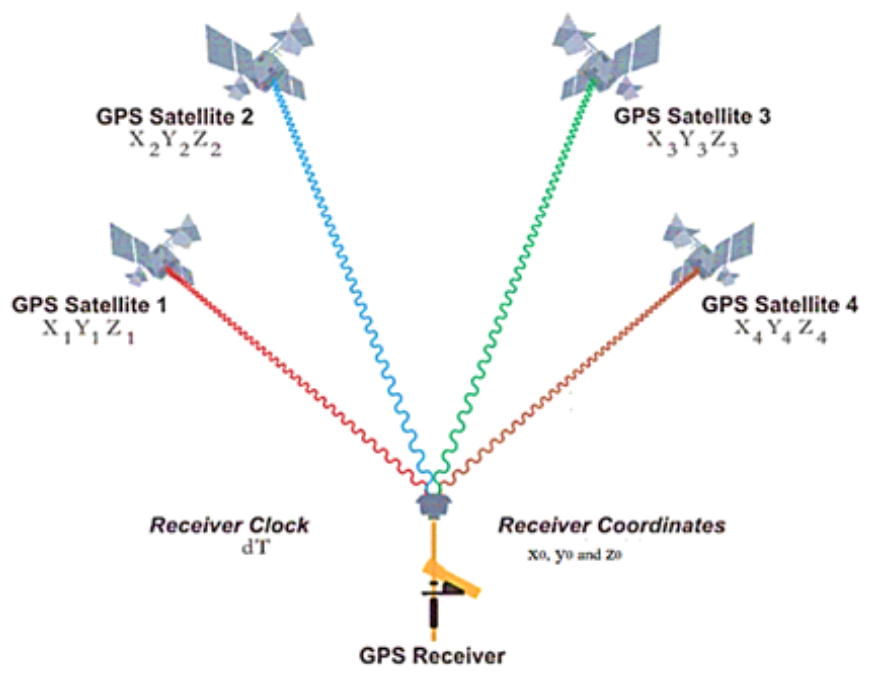

Figure 4: The GPS receivers on the earth with four satellites on view

\section{Google Map Locating}

Designers have developed a new technology in software applications that enable the subscriber to locate his position. Whenever the subscriber required to communicate with an application that gives a facility to association with geographic 
positions. This awareness equips different applications possibility to access to a specific location through Google map. Service of Google Map needs to specify API key for any operating system map before using this application. Google needs a Website registration and a decoding algorithm to extract the latitude and longitude data in a format script[2].

\section{Methodology}

Our methodology in the design consists of two phases, the software setup phase where all programs are needed to be installed in the embedded system and hardware setup phase including structuring and configuring the overall components of the system inside the vehicle as summarized by Fig. 5. For a beginning,RPi with Raspbian OS is associating. Notice the essential language here is a Python script that administering all configuration setup of the necessary modules. The procedures of communication between an embedded system and a vehicle's owner are performed as shown in the flowchart in Fig. 6. SMS reporting as a location coordinates is received to the owner for identified vehicles location, also MMS describing a driver's photo transmitted with high quality and better data rate over the internet. Every 1-minute vehicle's location SMS is updated and send with a new location. In the phase of the driver's image, there is a condition, If the driver is positively identified to the owner, the system is reset according to owner replied message. Now, if the condition was false the owner keeps tracking the location of a vehicle on Google map. When the owner decides to stop the vehicle at the specific position he sends an SMS to the vehicle that carries an instruction ordering the RPi to stop the vehicle.RPi forwarding this message as a high signal script to relay to switch off the engine and stop the vehicle.

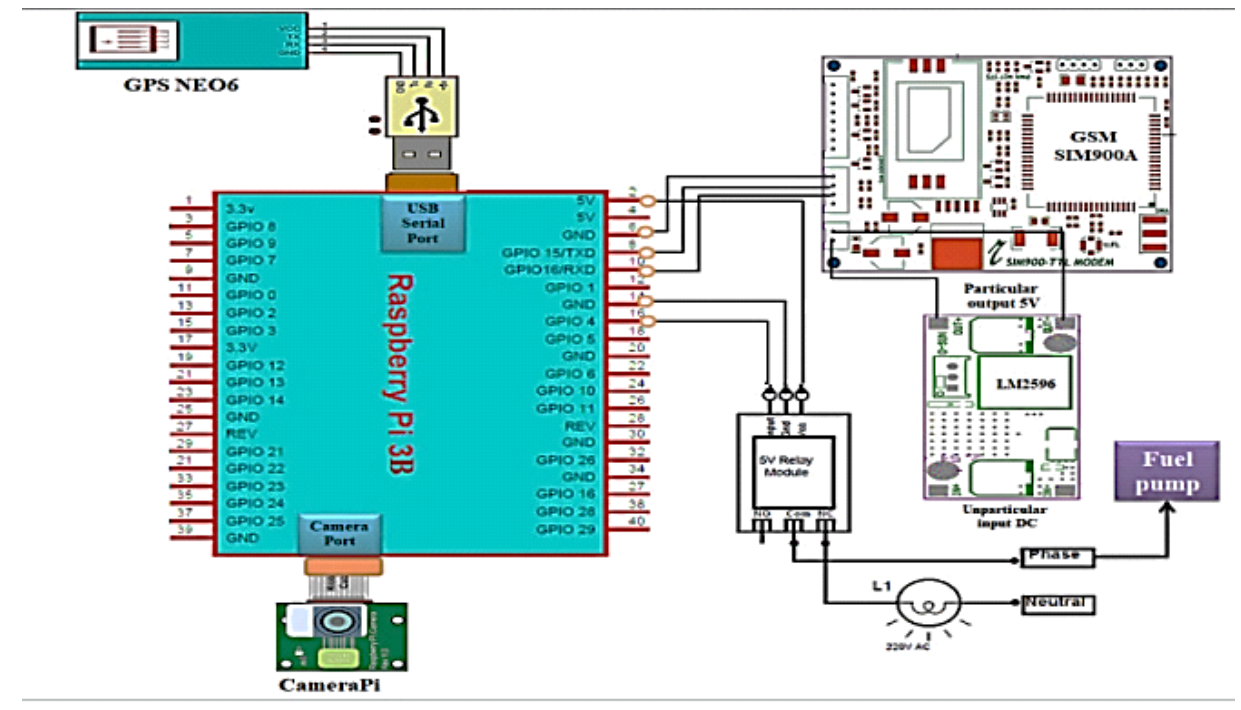

Figure 5: Block diagram of the system structure 


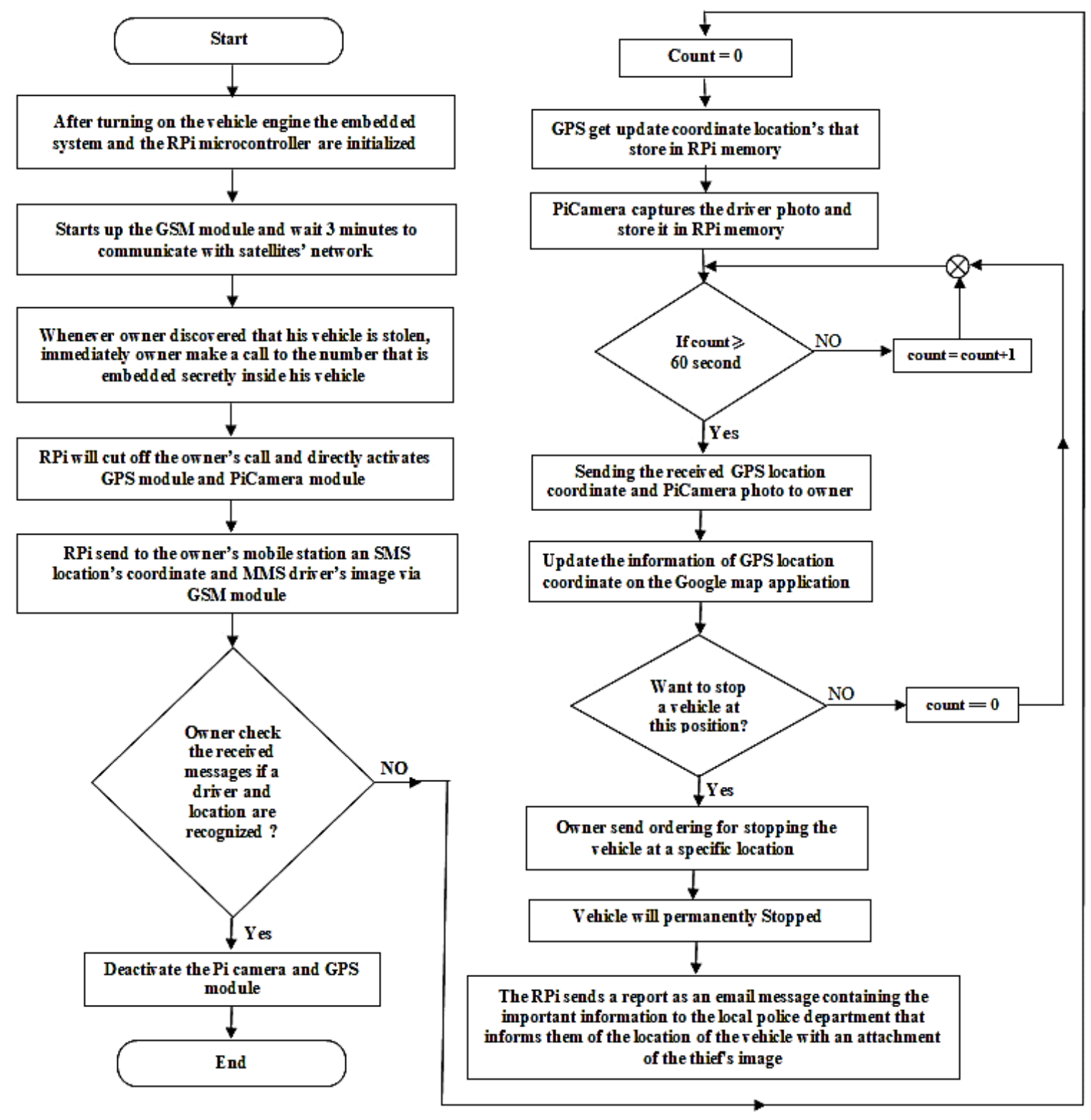

Figure 6: System flowchart

\section{RESULTS}

The integrated schematic diagram of the proposed system is shown in Fig. 7. The system functionality was examined using real data. The vehicle' location was using identification function and stored by applying real data. an evaluated process of the proposed system is fulfilled inside the vehicle's body that drives on a specific road in Baghdad with the privacy of the vehicle's owner. All examination units have correctly analyzed with positive results, in this investigation, 
two different locations were selected for testing in time. The results proved that the test system was classified successfully. Since there is no prior research applied raspberry pi in term of stopping the stolen vehicles.

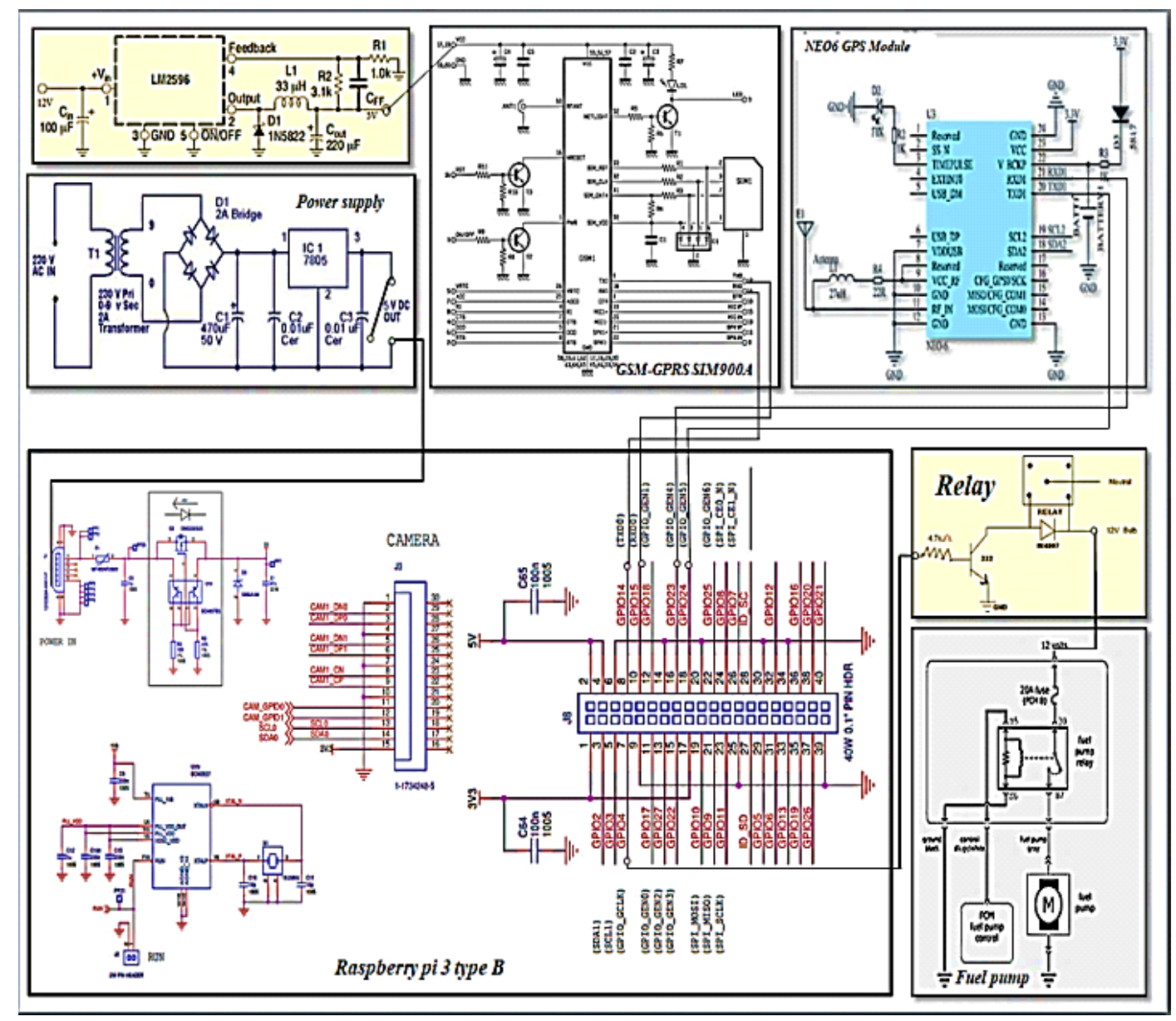

Figure 7: Schematic diagram for the proposed system

Fig. 8 shows the testing in case of receiving calling from GSM SIM900A module over "Minicom" application. This Figure also demonstrating how the module is faster, reliable and compatible with the RPi microcontroller. Also, Fig. 9 shows the streaming data of the identified location process in a specific location. It stored the location information in RPi memory. This figure illustrating the other necessary information received from the GPS module. Fig. 10, Fig. 11 representing the two locations coordinate in case of testing the running Python GPS program over RPi interface which appeared that the accuracy of our GPS module upon Linux Google earth is expected and acceptable. A red mark in Figures representing the vehicle location at a particular position where Fig. 11 points the same vehicle on the road with speed of $(15 \mathrm{~m} / \mathrm{s})$ after pass one minute from the previous location. The owner will continue to receive an update message for tracking process over Google map until a decision is demanded to stop the vehicle at the required position. 


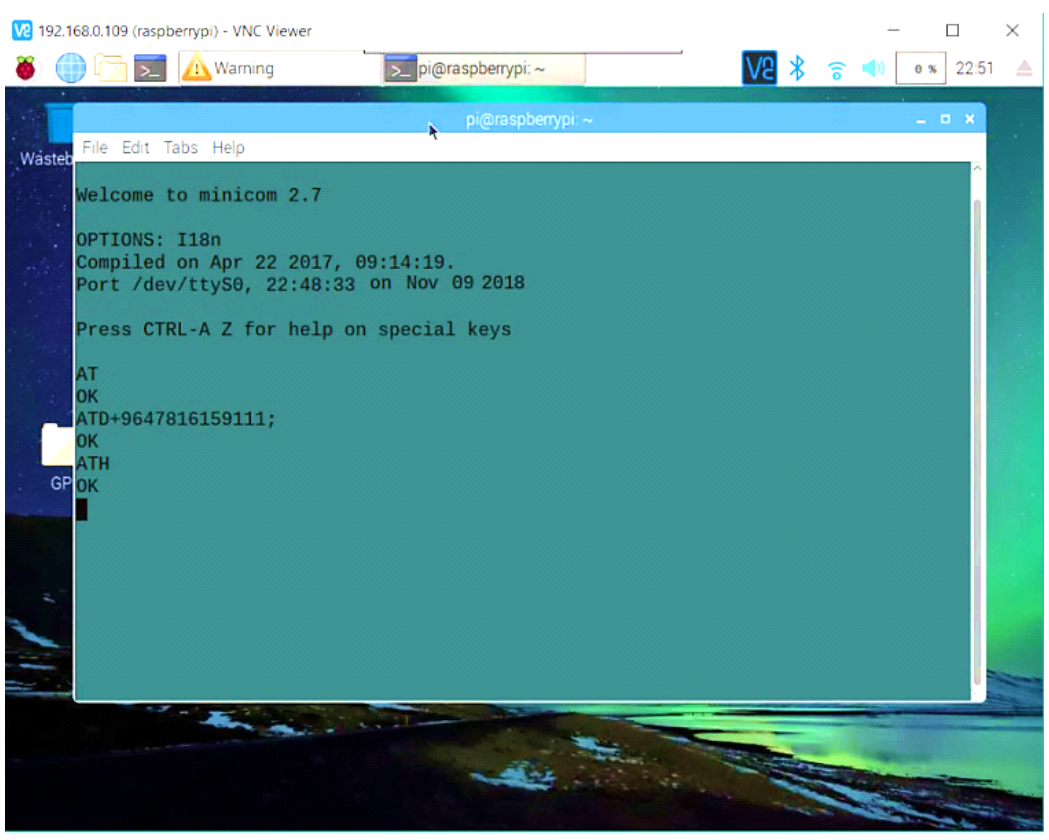

Figure 8: An output of received call over GSM module

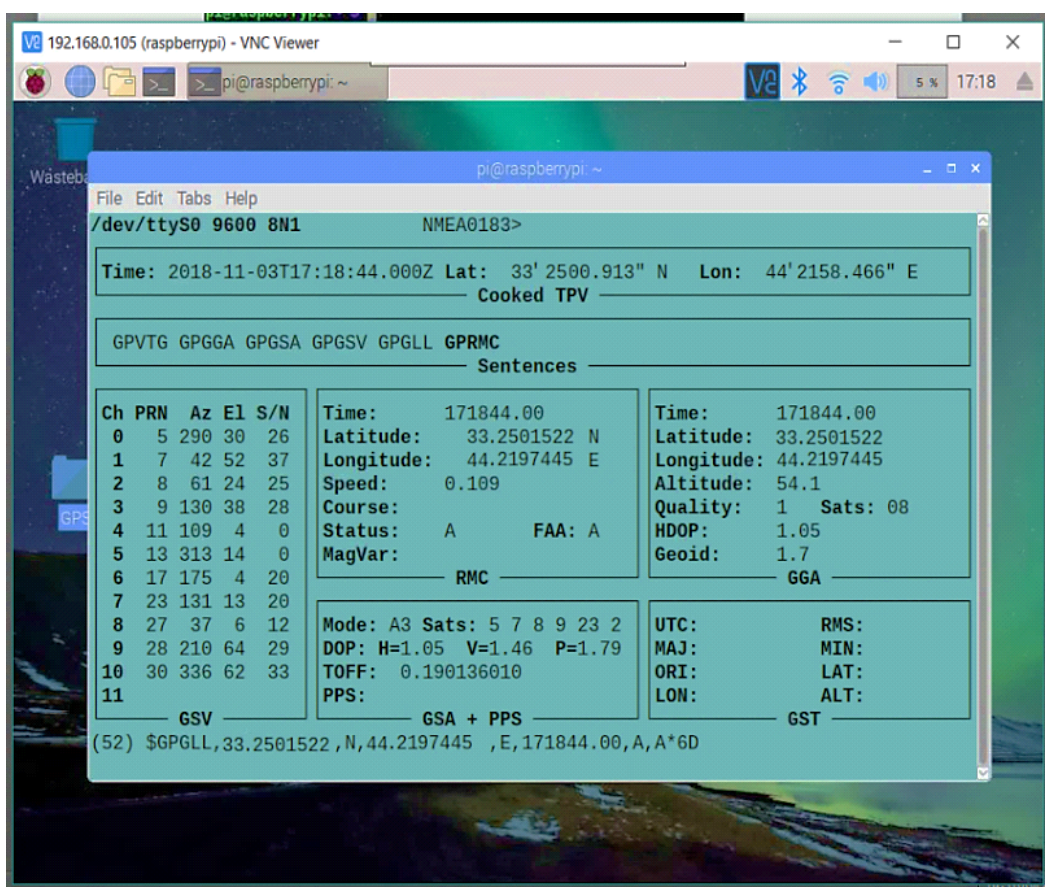

Figure 9: Output GPS location coordinate that store on RPi memory 


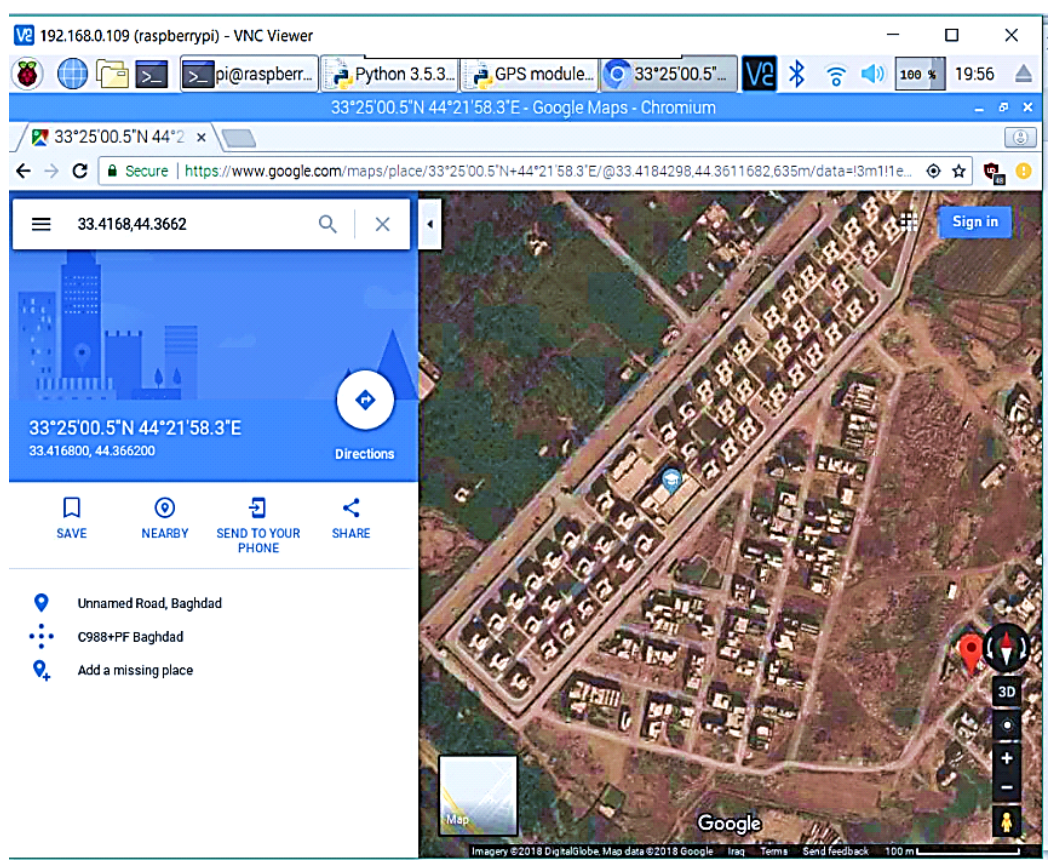

Figure 10: An output of tracking vehicle on the google map at specific location

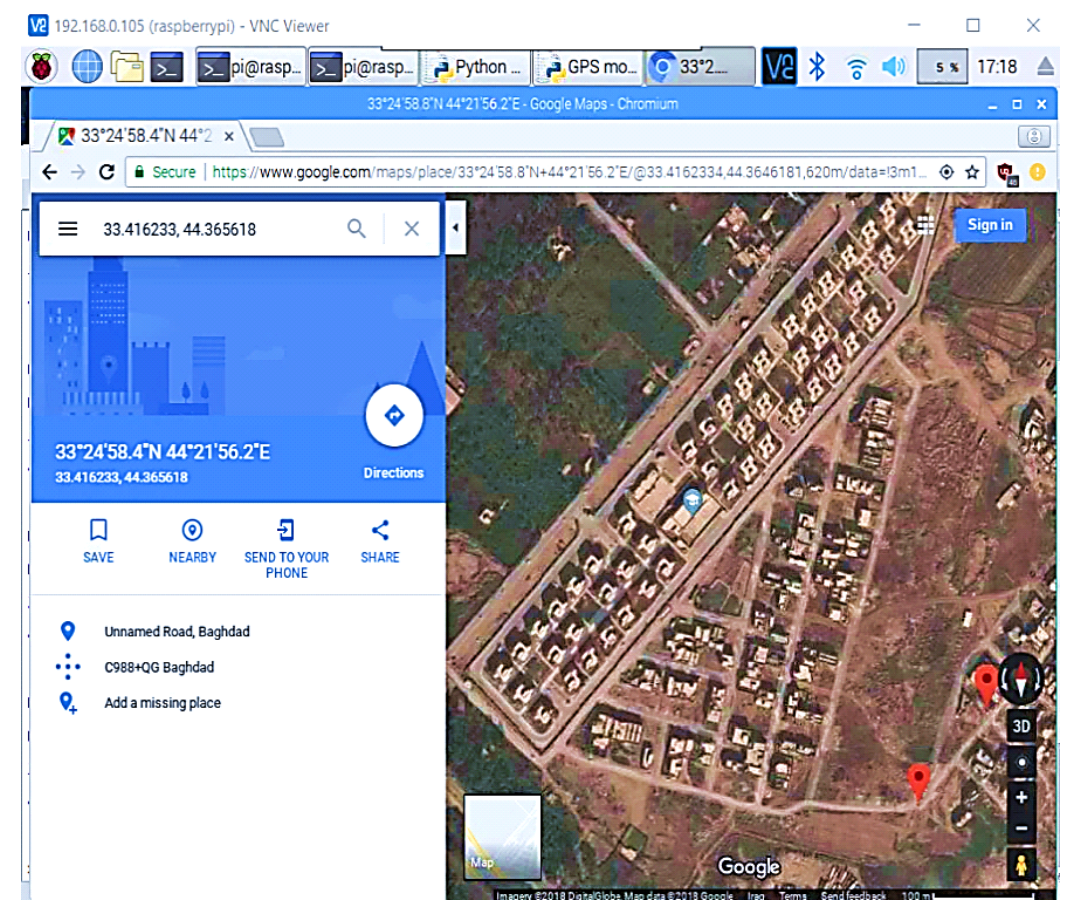

Figure 11: An output of tracking vehicle on the google map after 1-minute count

An SMS message that receiving to the owner with vehicle's locations is shown in Fig. 12 Whenever the owner press 
on that messages the Fig. 13,Fig. 14 will introduce the two different locations of the same vehicle according to SMS coordinates messages which are receiving from GSM and GPS of an embedded system inside the vehicle.

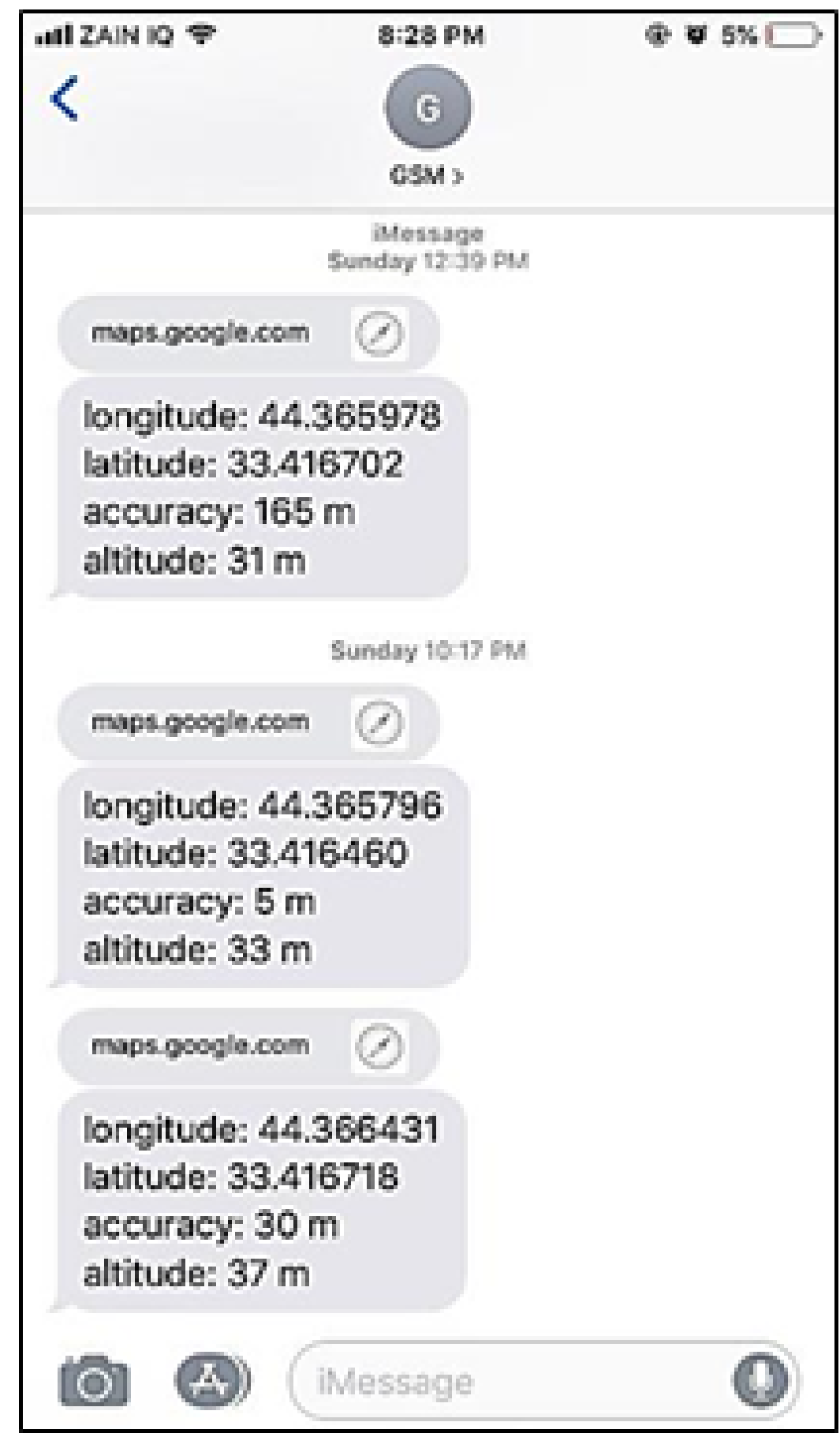

Figure 12: The current location of the vehicle and location of the Same vehicle after 1 minute 


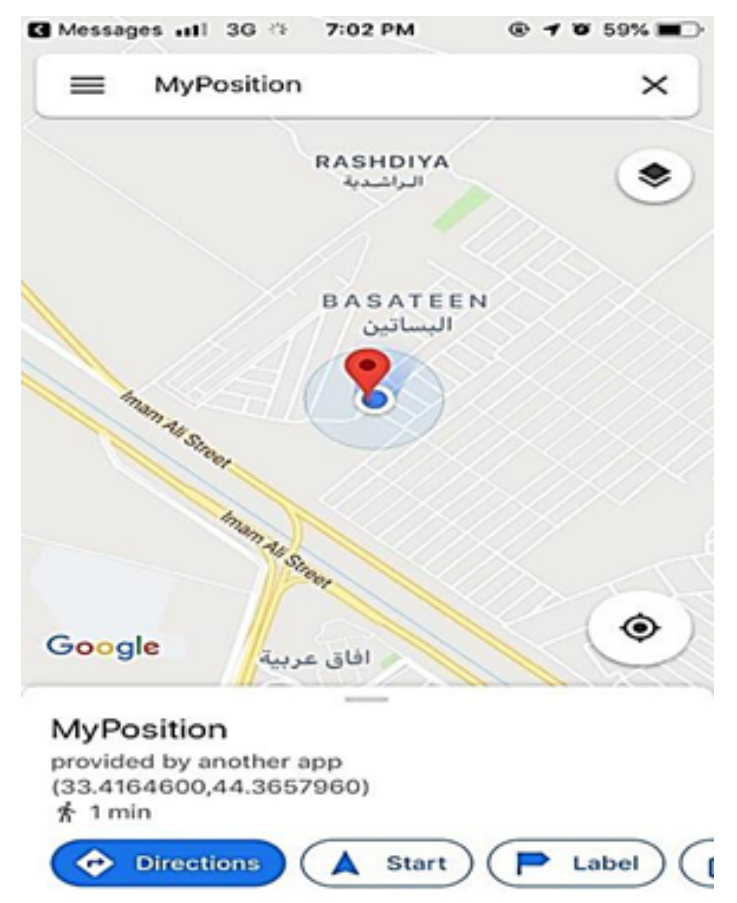

Figure 13: The output of tracking vehicle on the google map at specific location

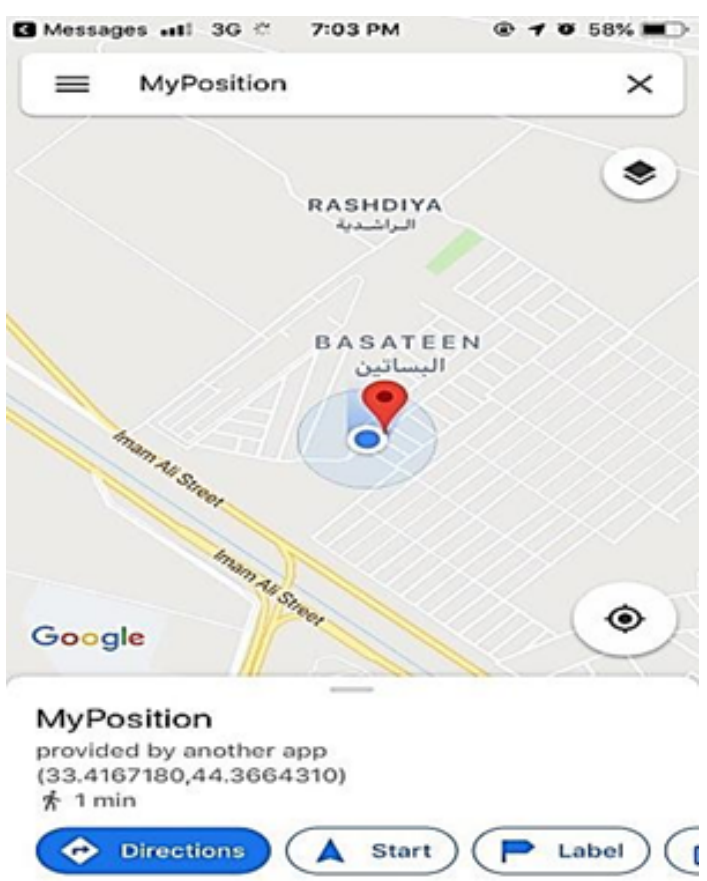

Figure 14: An output of tracking vehicle on the google map after 1-minute count 


\section{Conclusions}

An advanced vehicle anti-theft control system based on LTE Release 8 and Raspberry Pi microcontroller has been efficiently designed, and executed in this work. The project introduces a threat that is ultimately for vehicle thieves. The implementation of this project accomplished and characterized to perform the following points:

- It allows realizing and tracking the vehicle location over the Google map application.

- An advanced communication system has been used.

- The owner has full control to stop his vehicle at any specified location by controlling its engine remotely.

- All information related to the theft accident reported directly to a local police authority.

Higher quality and better data rate with exploiting services of the transmitted channel over the internet to capture a driver photo every 5 minutes maintained. The advanced system is designed to be at low cost and beyond 100\$. Evaluation tests show that the system is very effective in determining the location of the vehicle, identifying the driver and control the stopping of the vehicle at any preferred owner location along its root. If there is no Internet available for any reason, in this case, we will lose only the image of the driver where the subscriber cannot get it. An extended work in the future will solve this problem by letting an MMS facility to transfer the captured images to the owner.

\section{REFERENCES}

[1] Nagalaskhmi T S1, Nirmala L2 , and Akash Soragaon3, "Raspberry Pi based Embedded System for Vehicle Automation over Internet", International Journal of Advanced Research in Computer and Communication Engineering, Vol.5, Issue 12, ISSN (Online) 2278-1021, ISSN (Print)23195940, December 2016.

[2] Shruthi, K, Ramaprasad, P, Ray R, Naik, M.A, and Pansari, S, (2015,December), "Design of an anti-theft vehicle tracking system with a smartphone application", In Information Processing (ICIP), IEEE International Conference on(pp.755- 760) 2015.

[3] Liu, Z, Zhang, A and Li, S, (2013, July), "Vehicle anti-theft tracking system based on the Internet of things In Vehicular Electronics and Safety(ICVES)", IEEE International Conference on (pp. 48- 52) in 2013.

[4] Lee S, Tewolde, G, and Kwon, J, (2014, March), "Design and implementation of a vehicle tracking system using GPS, GSM, GPRS technology and smartphone application In the Internet of Things(WF-IoT)", IEEE World Forum on(pp. 353- 358), IEEE 2014.

[5] Shinde, Prashant A., and Y.B.Mane, "Advanced vehicle monitoring and tracking system based on Raspberry Pi Intelligent Systems and Control (ISCO)", 2015 IEEE 9th International Conference on IEEE, 2015.

[6] Ajish, T. , Fasil, M, Mohammed Shafeeq, K. K, Aswathi, P, and Leeshma, C. P "Raspberry Pi-Based Intelligent Car Anti-Theft System Through Face Recognition Using GSM and GPS", 2016, Available: https:/www.researchgate.net/profile/Mohammed-Shafeeq2/publication/326588033 Raspberry PiBased Intelligent Car Anti-Theft System Through Face Recognition Using GSM and GPS/links/Raspberry- Pi- Based- Intelligent- Car Anti Theft System Through Face-Recognition Using GSM and GPS.pdf

[7] Vaibhav Nalavade, et. al. "An Intelligent automobile Anti- Theft Tracking and Calamity Detection System Based on IoT using RASPBERRY PI For Real-Time Applications", International Journal of Advanced Research in Science and Engineering, vol NO. 06, Issue No. 11, ISSN 2319- 8354, in November 2017.

[8] Mohamad, O.A, Hameed, R. T, and Tapus, N, "Design and implementation of a real- time tracking system based on Arduino Intel Galileo", In Proceedings of 8th International Conference on Electronics, Computers and Artificial Intelligence(ECAI)(Vol. 8, No. 2), June 2016.

[9] Anandhalli M, Baligar VP, " A novel approach in real- time vehicle detection and tracking using Raspberry Pi", Alexandria engineering journal, 57(3):1597- 607, 1 Sep. 2018.

[10] https://www.geospatialworld.net/

[11] G. Jakeer Hussain1a and T. Srinivas Reddy2, "Advanced Anti-Theft ATM Security using Raspberry Pi", International Research Journal of Engineering and Technology, Volume. 03 Issue. 08, e-ISSN: 2395- 0056, p- ISSN: 2395- 0072 Aug. , 2016.

[12] Practical Raspberry Pi Projects Second Edition, 2016 Gordon and Gotch Australia Imagine Publishing Ltd.

[13] Schwartz, M. (2004), Mobile wireless communications, Cambridge University Press.

[14] Jain, S, Vaibhav, A, and Goyal, L, "Raspberry Pi based interactive home automation system through E-mail", In Optimization, Reliability, and Information Technology (ICROIT), IEEE International Conference on (pp. 277- 280), February 2014.

[15] https://www.researchgate.net/publication/320373039 Frequency Domain Multipath Fading Channel Simulator Integrated with OFDM Transmitter for E- UTRAN Baseband Traffic Generator/figures

[16] Ian F. Akyildiz, et. al ,"The evolution to 4G cellular systems: LTE- Advanced", Broadband Wireless Networking Laboratory, School of Electrical and Computer Engineering, Georgia Institute of Technology, Atlanta, GA 30332, United States, Elsevier B. V. All rights reserved, Physical Communication 217- 244, March (2010). 
[17] Abdullah, M, Abdullah, A. J, Ghanim, M, and Malaysia, H. O, "Comparison study on 3.9 G and 4G evolution", In International Conference on Information Communication and Management, IPCSIT (Vol. 16), 201.

[18] Gao, Mengdan, Tongyu Zhu, Xuejin Wan, and Qi Wang, "Analysis of travel time patterns in urban using taxi GPS data", In Green Computing and Communications (GreenCom), 2013 IEEE and Internet of Things (iThings/CPSCom), IEEE International Conference on and IEEE Cyber, Physical and Social Computing, pp. 512- 517, IEEE, 2013.

[19] Thompson, Richard B. , "Global positioning system: the mathematics of GPS receivers", Mathematics magazine 71, no. 4 (1998): $260-269$. 